\title{
Correction to: Genetics, cross-resistance and realized heritability of resistance to acetamiprid in generalist predator, Chrysoperla carnea (Steph.) (Neuroptera: Chrysopidae)
}

\author{
Muhammad M. Mansoor ${ }^{1,2^{*}}$ and Sarfraz A. Shad ${ }^{2^{*}}$
}

\section{Correction to: Egypt J Biol Pest Control (2020) 30:23 \\ https://doi.org/10.1186/s41938-020-0213-x}

Following publication of the original article (Mansoor and Shad 2020), the author's flagged that the article had published with two errors.

Firstly, part of the footnote of Table 1 was incorrect.

Secondly, the second author was not detailed as a cocorresponding author.

These errors have been corrected in the original article.

Furthermore, please find the corrected Table 1 and the corrected author list in this correction.

Published online: 04 May 2020

\section{Reference}

Mansoor, Shad (2020) Genetics, cross-resistance and realized heritability of resistance to acetamiprid in generalist predator, Chrysoperla carnea (Steph) (Neuroptera: Chrysopidae). Egypt J Biol Pest Control 30:23. https://doi.org/10. 1186/s41938-020-0213-x

The original article can be found online at https://doi.org/10.1186/s41938020-0213-x.

*Correspondence: honeybeepak@gmail.com; sarfrazshad@bzu.edu.pk

The original article can be found online at https://doi.org/10.1186/s41938020-0213-x

'Fatima Sugar Research \& Development Centre, Fatima Sugar Mills Ltd, Muzaffargarh, Punjab, Pakistan

${ }^{2}$ Department of Entomology, Faculty of Agricultural Sciences and

Technology, Bahauddin Zakariya University, Multan, Punjab, Pakistan

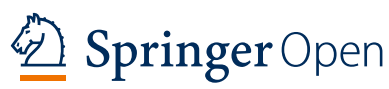

(๑) The Author(s). 2020 Open Access This article is licensed under a Creative Commons Attribution 4.0 International License, which permits use, sharing, adaptation, distribution and reproduction in any medium or format, as long as you give appropriate credit to the original author(s) and the source, provide a link to the Creative Commons licence, and indicate if changes were made. The images or other third party material in this article are included in the article's Creative Commons licence, unless indicated otherwise in a credit line to the material. If material is not included in the article's Creative Commons licence and your intended use is not permitted by statutory regulation or exceeds the permitted use, you will need to obtain permission directly from the copyright holder. To view a copy of this licence, visit http://creativecommons.org/licenses/by/4.0/. 
Table 1 Response of various insecticides to Lab-PK, Field, UNSEL and Aceta-SEL populations of Chrysoperla carnea

\begin{tabular}{|c|c|c|c|c|c|c|c|c|c|}
\hline \multirow[t]{2}{*}{ Strain } & \multirow[t]{2}{*}{ Insecticide } & \multirow[t]{2}{*}{$\mathrm{LC}_{50}(95 \% \mathrm{FL})\left(\mu \mathrm{gL}^{-1}\right)$} & \multicolumn{4}{|c|}{ Fit of probit line } & \multirow[t]{2}{*}{$N^{a}$} & \multirow[t]{2}{*}{$R^{\mathbf{b}}$} & \multirow[t]{2}{*}{$\mathrm{RR}^{c}$} \\
\hline & & & Slope $( \pm$ SE) & $x^{2}$ & df & $P$ & & & \\
\hline \multirow[t]{4}{*}{ Lab-PK (G130) } & Acetamiprid & $0.72(0.45-0.95)$ & $2.26(0.36)$ & 1.48 & 3 & 0.86 & 350 & 1 & \\
\hline & Spinosad & $1.15(0.94-1.32)$ & $3.21(0.40)$ & 0.46 & 3 & 0.98 & 350 & 1 & \\
\hline & Buprofezin & $4.27(3.34-5.10)$ & $2.68(0.35)$ & 1.88 & 3 & 0.51 & 350 & 1 & \\
\hline & Pyriproxyfen & $5.54(4.53-6.50)$ & $2.55(0.30)$ & 4.13 & 3 & 0.63 & 350 & 1 & \\
\hline \multirow[t]{4}{*}{ Field (G1) } & Acetamiprid & 1676.07 (1171.58-2867.68) & $1.32(0.26)$ & 1.46 & 3 & 0.69 & 350 & 2327.87 & \\
\hline & Spinosad & 877.29 (607.27-1547.13) & $1.31(0.27)$ & 0.71 & 3 & 0.87 & 350 & 762.86 & \\
\hline & Buprofezin & 113.39 (82.96-160.79) & $1.48(0.26)$ & 5.74 & 3 & 0.12 & 350 & 26.55 & \\
\hline & Pyriproxyfen & 335.77 (274.89- 418.66) & $1.68(0.22)$ & 1.06 & 3 & 0.79 & 350 & 60.61 & \\
\hline \multirow[t]{4}{*}{ UNSEL (G16) } & Acetamiprid & $133.10(111.35-158.30)$ & $2.00(0.23)$ & 1.72 & 3 & 0.63 & 350 & 184.86 & \\
\hline & Spinosad & 75.40 (57.90-91.26) & $2.24(0.28)$ & 1.52 & 3 & 0.68 & 350 & 65.56 & \\
\hline & Buprofezin & $16.42(13.68-18.97)$ & $2.94(0.34)$ & 2.45 & 3 & 0.48 & 350 & 3.84 & \\
\hline & Pyriproxyfen & $162.53(138.76-186.15)$ & $2.89(0.30)$ & 3.80 & 3 & 0.28 & 350 & 29.33 & \\
\hline \multirow[t]{4}{*}{ Aceta-SEL (G16) } & Acetamiprid & 22370.54 (12089.78-99622.35) & $1.25(0.28)$ & 0.30 & 3 & 0.82 & 350 & 31070.19 & 13.34 \\
\hline & Spinosad & 1531.44 (953.33-4323.61) & $1.12(0.23)$ & 1.26 & 3 & 0.99 & 350 & 1331.69 & 1.75 \\
\hline & Buprofezin & $342.52(221.09-829.50)$ & $1.38(0.26)$ & 0.92 & 3 & 0.74 & 350 & 80.21 & 3.02 \\
\hline & Pyriproxyfen & $1322.92(857.01-3174.71)$ & $1.35(0.27)$ & 1.62 & 3 & 0.65 & 350 & 238.79 & 3.94 \\
\hline
\end{tabular}

${ }^{a} \mathrm{~N}$ is number of total larvae exposed in bioassay including control

${ }^{\mathrm{b}} \mathrm{RR}$ resistance ratio, $\mathrm{LC}_{50}$ of Field or UNSEL or Aceta-SEL / $\mathrm{LC}_{50}$ of Lab-PK strain

${ }^{\mathrm{C}} \mathrm{RR}$ resistance ratio, $\mathrm{LC}_{50}$ of Aceta-SEL / LC $\mathrm{C}_{50}$ of Field strain 